\section{Return of a normal functioning spleen after traumatic splenectomy}

\section{Z A J Khan MS FRCS P E Dikki FRCS}

J R Soc Med 2004;97:391-392

Splenectomy renders a patient susceptible to infection by encapsulated bacteria and calls for long-term antibiotic prophylaxis. But splenic function can return.

\section{CASE HISTORY}

A man of 54 was admitted with severe lower abdominal pain, of sudden onset and spasmodic in nature, for the past twenty hours. His medical history included a total splenectomy after splenic trauma 20 years before, since when he had been taking penicillin $250 \mathrm{mg}$ twice daily. On examination he was febrile (temperature $37.8^{\circ} \mathrm{C}$ ) and tachycardic. The abdomen was slightly distended and tender with guarding in the left iliac fossa. Apart from a raised white cell count $\left(14.2 \times 10^{-9} / \mathrm{L}\right)$ haematological and biochemical indices were normal. A plain supine abdominal radiograph on admission showed normal calibre small and large bowel. The provisional diagnosis was diverticulitis and he was started on intravenous antibiotics. A CT scan suggested a pelvic mass overlying the bladder, but also showed a lobulated spherical mass under the left hemidiaphragm with appearance and size suggestive of spleen. A fibreoptic examination was limited to the rectosigmoid because of inability to negotiate the lumen any further. A water-soluble contrast enema displayed a sigmoid stricture which allowed very little contrast to pass through.

Because of unsettled pain, a laparotomy was undertaken through the previous midline splenectomy scar, and the sigmoid mass was excised with en-bloc resection of involved bladder. Histological examination confirmed complicated diverticular disease. A full-size spleen was obvious under the left hemidiaphragm.

\section{COMMENT}

Spleen can regenerate through various mechanisms. Autotransplantation of splenic tissue after traumatic disruption of the splenic capsule is well recognized. ${ }^{1}$

Department of Surgery, Queen Elizabeth Hospital, King's Lynn PE30 4ET, UK

Correspondence to: Z A J Khan\#E-mail: zulfiqarajkhan@hotmail.com
Splenic tissue can lodge anywhere in the peritoneal cavity following traumatic disruption and regenerates under favourable conditions. ${ }^{2}$ The incidence of splenic regeneration correlates with the severity of splenic injury: ${ }^{3}$ patients requiring a splenectomy for trauma tend to be those with greatest splenic damage and dissipation of splenic tissue, which favours autotransplantation. These splenic implants, called splenosis, can be found anywhere in the peritoneal cavity. They are supplied by newly formed arteries that penetrate the capsule. ${ }^{4}$

Accessory spleens, on the other hand, are common prevalence $10-31 \%$ in autopsy series. ${ }^{5}$ They result from incomplete fusion of separated fetal spleen tissue originating from the dorsal mesogastrium. ${ }^{6}$ In healthy individuals, accessory spleens usually measure only a few millimetres in diameter ${ }^{5}$ and may be undetectable on routine imaging. These accessory spleens can enlarge following splenectomy and be the source of recurrent symptoms in those operated on for blood disorders. An accessory spleen derives its blood supply from branches of the splenic artery. ${ }^{4}$

When a surgeon deals with a bleeding spleen in an emergency, some splenic tissue may remain in situ at the hilum. We suspect that this was the origin of our patient's neoformed spleen, because of its normal anatomical position and blood supply from splenic vessels. ${ }^{7}$

Overwhelming sepsis from encapsulated bacteria is a major complication of splenectomy. ${ }^{8}$ The risk of subsequent infection is lowest with traumatic splenectomy, ${ }^{9}$ possibly because function can return. The notion that regenerated spleens have a functional value is supported by their normal tissue architecture. ${ }^{1}$ More importantly, the volume of regenerated splenic tissue seems to correlate with protection against postsplenectomy infection. ${ }^{10}$

The existence of functional splenic tissue is indicated by the absence of Howell-Jolly bodies and presence of normal IgM blood levels. ${ }^{8}$ These were the findings in our patient, and the prophylactic penicillin was stopped. Some immunologists, to be on the safe side, would also wish to see normal $\operatorname{IgG}$ levels to pneumococcal polysaccharides.

\section{REFERENCES}

1 Mostbeck G, Sommer G, Haller J, et al. Accessory spleen: presentation as a large abdominal mass in an asymptomatic young woman. Gastrointest Radiol 1987; 12:337-9

2 Orda R, Barak B, Baron J, Spirer Z, Wiznitzer T. Postsplenectomy splenic activity. Ann Surg 1981;194:771-4

3 Muller U, Rothlin M. Splenic neoformation following trauma-induced splenectomydiagnosis and function. Swiss Surg 1995;5:230-5

4 Bertolotto M, Gioulis E, Ricci C, Turoldo A, Convertino C. Ultrasound and Doppler features of accessory spleens and splenic grafts. Br J Radiol $1998 ; 71: 595-600$

5 Curtis GM, Movitz D. The surgical significance of accessory spleen. Ann Surg 1946;123:276-98 
6 Dodds WJ, Taylor AJ, Erickson SJ, Stewart ET, Lawson TL. Radiologic imaging of splenic anomalies. Am $J$ Roentgenol 1990; 155:805-10

7 Revuelta Alvarez S, Fernandez-Escalante C, Casanova Rituerto D, Lopez Espadas F, Martin Fernandez J. Assessment of post-splenectomy residual splenic function. Splenic autotransplants. Int Surg 1987;72:149-53

8 Sumaraju V, Leon G, Smith SM. Infectious complications in asplenic hosts. Infect Dis Clin N Am 2001;15:551-65

9 Sherman R. Perspectives in management of trauma to spleen: 1979 presidential address, American Association for the Surgery of Trauma. J Trauma 1980;20:1-13

10 Zoli G, Corazza GR, D’Amato G, Bartoli R, Baldoni F, Gasbarrini G. Splenic autotransplantation after splenectomy: tuftsin activity correlates with residual splenic function. Br J Surg 1994;81:716-18

\section{Pulmonary embolism caused by Candida albicans}

\author{
J Villaquiran $M^{1} \quad S$ M Allen FRCS ${ }^{1}$ \\ A J Marshall MD FRCP ${ }^{2} \quad \mathrm{~N} J$ Ring FRCR $^{3}$
}

J R Soc Med 2004;97:392-393

Native valve infective endocarditis usually affects the mitral or aortic valve and the incidence of systemic embolization is about 20\%. Fungal endocarditis, which accounts for $1 \%$ of cases, has special features.

\section{CASE HISTORY}

A man aged 72 was admitted after becoming increasingly short of breath in the past week, much worse over the past two days. On the day of admission he had had a cough and some haemoptysis. Eleven months previously he had had an anterior resection for rectal carcinoma and had subsequently required readmission with signs of peritonitis and renal failure. At laparotomy the anastomosis was found to have broken down with faecal peritonitis and a Hartmann's procedure was performed. He had a protracted and stormy course in the intensive therapy unit with multiple episodes of sepsis including meticillin-resistant Staphylococcus aureus, pseudomonas and lastly Candida albicans in blood cultures. He eventually recovered, but four weeks before the current admission an isotope ventilation and perfusion scan, requested by his general practitioner, had shown a high probability of pulmonary emboli (Figure 1) and he had been started on anticoagulants.

Departments of ${ }^{1}$ Cardiothoracic Surgery, ${ }^{2}$ Cardiology, ${ }^{3}$ Radiology, Derriford Hospital, Plymouth, Devon PL6 8DH, UK
On examination he was dyspnoeic with slight bilateral pedal oedema and a raised jugular venous pressure. Oxygen saturation was $69 \%$ on air and the provisional diagnosis was recurrent pulmonary embolism; this became less likely when the international normalized ratio was reported as 5.3. He remained very short of breath, and an echocardiogram three days after admission showed a large vegetation on the tricuspid valve, $2.2 \mathrm{~cm}$ in diameter (Figure 2). There was mild dilatation of the right atrium and ventricle and pulmonary artery pressure was considerably above normal (about $60 \mathrm{mmHg}$ ). A CT pulmonary angiogram then revealed large filling defects in the artery to the left lower lobe and also filling defects in both upper lobe arteries (Figure 3). Subsequently, blood cultures yielded $C$. albicans identical to the strain isolated during the patient's previous stay in the intensive care unit. Flucytosine and amphotericin were prescribed.

At operation the chest was opened through a median sternotomy and pulmonary artery pressures were found to be about two-thirds of systemic. Full cardiopulmonary bypass was established with ascending aortic return and bicaval venous drainage. The anterior and septal leaflets of the tricuspid valve proved to be completely covered with fungal growth. The valve was partly excised and reconstructed. Large amounts of embolic fungal material were removed by means of a standard embolectomy catheter from the left lower and right upper lobe arteries and a smaller amount from the left upper pulmonary artery. Subsequent cultures from the tricuspid valve confirmed the presence of $C$. albicans. The antifungal regimen was continued and the patient was discharged six weeks after admission.

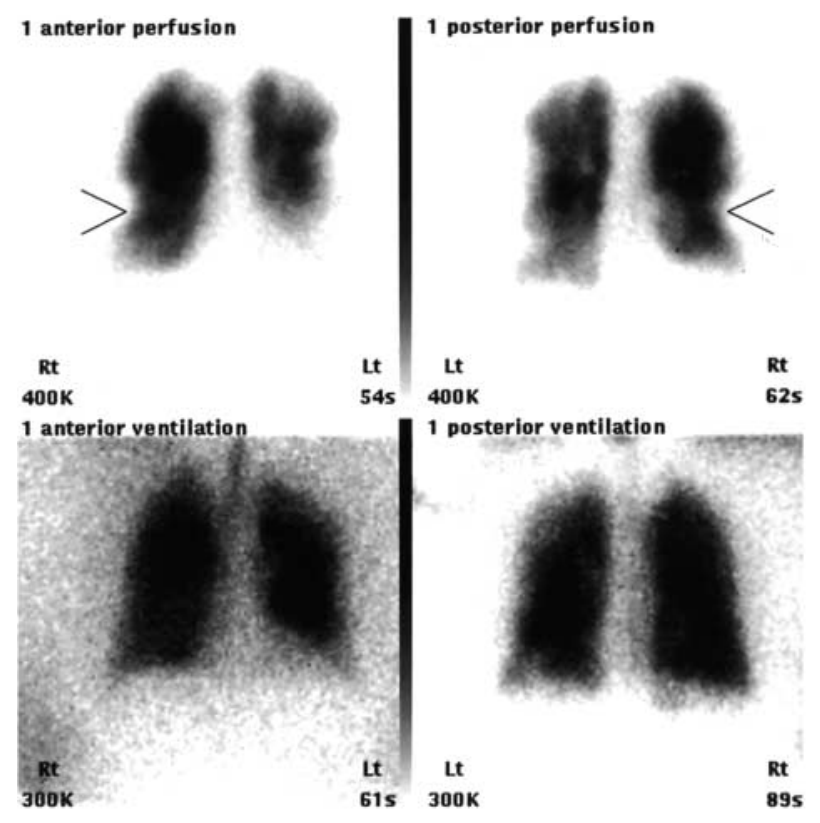

Figure 1 VQ scan showing multiple defects on perfusion images (arrows) with normal ventilation 